\title{
A practical and transferable new protocol for treadmill testing of children and adults?
}

Received: 6 February 2009; Accepted: 4 May 2009; First published online: 7 October 2009

Sir,

The article of Dubowy et al. ${ }^{1}$ provides reference values for maximal exercise performance evaluated by a simple treadmill test in a population ranging from 4 to 75 years of age, classified according to gender and age both with and without relation to body mass and body surface area. The purpose deserves attention, especially in view of the increasing group of adults with congenital cardiac disease, whose exercise capacity requires a comparison with normal values.

It is, however, questionable whether the population chosen for comparison by the authors meets the demands to be claimed for normal controls. No information is given regarding the attitude of the participants towards physical activity or sports, their social classification, whether the subjects comprised entire classes of kindergardens, schools or facilities for adult education, or whether they represented selected members. The latter is to be assumed, since the participants were "volunteers", signifying self-selection. A classical publication relevant to the topic ${ }^{2}$ demonstrates in exemplary fashion the importance of the kind of provision of control values. Maximal treadmill tests were used to assess the cardiovascular performance capacity of children with insignificant cardiac defects. When compared to controls seen in the same clinic because of innocent cardiac murmurs, the patients proved to be normal. When volunteers selected from schools were taken for comparison, even children with the most trivial defects showed distinct limitations. The conclusion drawn by the author was that volunteers represent a self-selected population unsuitable for provision of standard values.

The values of body measurements of the probands summarily provided by the authors permit no

Correspondence to: Prof. Dr Rolf Mocellin, Höllgrub 88, 84367 Zeilarn, Germany. Tel: +49 8678 571; Fax: +49 8678 986948; E-mail: mocellin@ t-online.de conclusion as to the representativeness of the population examined. There are no statements concerning the number of probands and the corresponding values of body size in different age groups, which, however, must be regarded indispensable for critical evaluation with regard to magnitude and distribution especially in children and adolescents because of the rapid changes present during growth.

Doubts concerning the representativeness of the measurements are confirmed by the surprising finding of a constant increase of peak oxygen uptake related to body mass during adolescence in the male probands. Peak oxygen uptake has been expressed relative to total body mass to account for differences in body size by many authors, ${ }^{3,4,5}$ and the picture that has emerged shows that peak oxygen uptake in boys remains remarkably consistent, at least over the range of 8 to 18 years.

Besides, when standard values of physical fitness are to be evaluated, the question arises as to what reference parameters the absolute values should be related to. Dubowy et al. ${ }^{1}$ rely on body mass, thus admitting an undue significance to the fat component of the body, as lean body mass is not at hand. The use of body mass as a reference parameter for cardiovascular performance may thus turn out to be a method that, indirectly, estimates overweight. Similarly, body surface area is of limited value in individuals, the more as it cannot be measured directly. Therefore, the square of body height in metres should be considered the more appropriate reference parameter for standard values of peak oxygen uptake. As older adolescents are generally fitter than younger ones of the same height, age should be considered simultaneously with height ${ }^{6}$, in analogy to how Dubowy et al. ${ }^{1}$ have done with age and body mass.

$$
\begin{array}{r}
\text { Rolf Mocellin } \\
\text { Department of Pediatric Cardiology } \\
\text { University of Freiburg } \\
\text { Germany }
\end{array}
$$




\section{References}

1. Dubowy KO, Baden W, Bernitzki S, Peters B. A practical and transferable new protocol for treadmill testing of children and adults. Cardiol Young 2008; 18: 615-623.

2. Cumming GR. Maximal exercise capacity of children with heart defects. Am J Cardiol 1978; 42: 613-619.

3. Astrand PO. Experimental studies of physical working capacity in relation to sex and age. Ejnar Munksgaard, Copenhagen, 1952.
4. Máček M, Čermak V, Handzo P, Horák J, Jirka Z, Rouš J, Seliger V, Ulbrich J. Comparison between 12, 15 and 18 year old groups of country and urban boys and girls. In: Seliger V (ed.). Physical fitness. Universita Karlova, Praha, 1973, pp 244-247.

5. Armstrong N, Welsman JR. Peak oxygen uptake in relation to growth and maturation in 11 - to 17 - year - old humans. Eur J Appl Physiol 2001; 85: 546-551.

6. Mocellin R. Exercise testing in pediatric cardiology. In: Torg JS, Welsh RP, Shephard RJ (eds). Current therapy in sports medicine- 2. BC Decker, Philadelphia, 1990, pp 34-39. 